\title{
The Area of Pressure-Induced Referred Pain Is Dependent on the Intensity of the Suprathreshold Stimulus: An Explorative Study
}

Palsson et al.

Pain Referral Depends on Stimulation Intensity

\section{Thorvaldur Skuli Palsson, PhD ${ }^{1}$ Shellie Ann Boudreau, PhD $^{2}$ María Ortiz Lucas, PhD $^{3}$ Elisabeth Bravo Esteban, PhD ${ }^{4}$ Miriam Garrigós-Pedrón, PhD ${ }^{6}$ Pablo Herrero, PhD ${ }^{3}$ and Víctor Domé- nech-García, $\mathrm{MSc}^{3}$ [AQ1]}

1. Department of Health Science and Technology, SMI, Faculty of Medicine, Aalborg University, Aalborg, Denmark

2. Center for Neuroplasticity and Pain (CNAP), Department of Health Science and Technology, SMI, Aalborg University, Aalborg, Denmark

3. Universidad San Jorge, Zaragoza, Spain

4. Toledo Physiotherapy Research Group (GIFTO), E.U.E. Fisioterapia de Toledo, Universidad de Castilla la Mancha, Spain [AQ2]

5. Departamento de Fisioterapia, Motion in Brains Research Group, Institute of Neuroscience and Sciences of the Movement (INCIMOV), Centro Superior de Estudios Universitarios La Salle, Universidad Autónoma de Madrid, Madrid, Spain

6. Departamento de Ciencias Biomedicas, Facultad de Ciencias de la Salud, Universidad Cardenal Herrera-CEU, CEU Universities, Alfara del Patriarca, Valencia, Spain

Correspondence to: Thorvaldur Skuli Palsson, PhD, Department of Health Science and Technology, SMI, Faculty of Medicine, Aalborg University, Fredrik Bajers Vej 7D-3, Aalborg E 9220, Denmark. Tel: 45-9940-7518; Fax: 45-9815-4008; E-mail:

tsp@hst.aau.dk. [AQ3]

Disclosure: None of the authors has received any form of financial support in relation to this article. Shellie Ann Boudreau has holdings in Aglance Solutions ApS (Denmark), which licenses an online version of Navigate Pain. All other authors declare no conflicts of interest.

\section{ABSTRACT}

To investigate the pain referral area (number of pixels) and extent (vector length) as elicited from increasing intensities of pressure-induced pain at the shoulder.

Cross-sectional design.

Clinical laboratory setting.

Twenty-two healthy men and women participated in two experimental sessions.

Delayed onset of muscle soreness (DOMS) was induced in the dominant shoulder and assessed 24 hours later. Participants rated the level of DOMS on a 6-point Likert scale. Four different intensities (pressure pain threshold [PPT]+20\%, PPT+30\%, PPT+40\%, and PPT+50\%) were applied to the infraspinatus in a randomized, balanced fashion for 60 seconds from low to high intensity or vice versa. The resulting location, area, and extent of referred pain as drawn by the participants on a digital body chart were extracted and expressed in pixels. The extent of pain was defined as the vector length extending from the ipsilateral earlobe to the most distal location of the pain.

The referred pain area from PPT+20\% was smaller than PPT+30\%, PPT+40\%, and PPT+50\%. The extent of referred pain did not differ between the pressure pain intensities.

Pressure intensity at PPT $+30 \%$, but no more, produces the greatest referred pain area as compared with the traditional pressure intensity of PPT $+20 \%$. Thus, the intensity of PPT $+30 \%$ may be ideal for exploring the mechanisms of referred pain. The extent of the pain represents an independent expression of the intensity of the provoking stimulus and may be more closely related to the location of the stimulus.

Keywords: Referred Pain ; Suprathreshold Pressure Stimulation ; Digital Pain Mapping 


\section{Introduction}

Referred pain is a cardinal symptom in musculoskeletal pain, defined as pain felt remote to the site of nociceptive activity [1]. The phenomenon is commonly seen in clinical practice [2-4] and is reproducible in experimental settings $[5,6]$. An expansion in the area of referred pain, as assessed in somatic structures, may reflect increases in the sensitivity of central pain mechanisms $[4,7,8]$ and may as such be an interesting parameter to evaluate in research and clinical practice [AQ4].

Referred pain is often induced by injecting chemical agents (e.g., hypertonic saline or capsaicin) into somatic structures [9]. This may, however, not be feasible due to the invasive nature of the procedure and calls for alternative methods for inducing referred pain. In that respect, applying a tonic, nociceptive pressure stimulus on somatic structures is known to effectively induce referred pain in healthy individuals $[7,8,10,11]$. Furthermore, sensitizing the area by way of, for example, inducing exercise-related muscle soreness (delayed onset of muscle soreness [DOMS]) prior to stimulating it results in a pain referral pattern of a greater area [12], resembling what is seen following a hypertonic saline injection $[7,13]$. Considering that pain referral seems to be facilitated in persons suffering from chronic pain $[4,12]$ and in people who have recovered from a musculoskeletal injury [10, 14], inducing muscle soreness is a surrogate pain model to investigate referred pain.

Previous studies have shown that although suprathreshold pressure stimulation can induce referred pain, the pain area from, for example, hypertonic saline is larger [7, 10]. This may relate to a difference in stimulation intensity [10], and therefore it is possible that stimulating with greater pressure will result in a facilitated pain referral pattern. However, although the [AQ5] parameters of hypertonic saline stimulation (e.g., volume, concentration) are well established [9], the optimal parameters for pressure stimulation are not known. Previous studies have used stimulation intensities at both $20 \%$ [7] and 30\% [8] above the pressure pain threshold (PPT). In this regard, it is unclear whether increasing the stimulation linearly increases the pain referral pattern expressed as the total area of pain or the distance (extent) from the stimulus. From an ethical point of view, however, it is important that the research tools provide as much information as possible while causing the research participant as little discomfort as possible.

The objective of this explorative study is to compare the area and extent of referred pain as elicited by using standardized increases in the PPT. We hypothesized that increasing pressure intensities would elicit stepwise increases in the area of referred pain and the extent of the pain.

\section{Methods}

A convenience sample of 22 healthy, pain-free participants ( 10 nonpregnant women) were recruited from a university population. The sample size was based on a previous protocol [7] in which an expansion of pain referral was demonstrated from a sore shoulder muscle in otherwise healthy individuals. To be eligible, participants needed to be over 18 years with no current or previous history of an ongoing pain condition and/or any other disease. Moreover, any current or previous use of prescription medication or other substances that might affect the sensory system at the time of the study was an exclusion criterion. Nursing and pregnant women were also excluded. Participants were included after providing informed consent and were blinded to the expected outcome of the study. The study was approved by the local ethics committee (C.P.-C.I. PI16/0253), conformed to the Helsinki Declaration, and adhered to the STROBE statement for observational studies.

\section{Protocol}

This cross-sectional study was conducted in two experimental sessions 24 hours apart. All data were collected in a closed and quiet clinical laboratory setting with a stable room temperature. In the first session, participants performed eccentric exercise for the shoulder's external rotators to induce DOMS. In the second session, participants rated their DOMS level on a 0- to 6-point Likert scale. Pressure pain sensitivity was measured at the infraspinatus of the DOMSaffected shoulder using a handheld pressure algometer. Subsequently, a painful pressure stimulation was performed at four different suprathreshold stimulation intensities (PPT $+20 \%$, PPT $+30 \%$, PPT $+40 \%$, and PPT $+50 \%$, respectively), and the pain area following the stimulation was drawn on the posterior side of a digital body chart. 


\section{Delayed-Onset Muscle Soreness}

DOMS was induced prior to applying painful pressure, as it results in a greater frequency of referred pain from suprathreshold stimulation in healthy individuals [7,11]. DOMS was induced in the dominant shoulder by way of eccentric exercise until failure. The protocol for the exercise has previously been used for inducing soreness in the shoulder [7]. When performing the exercise, the participant sat upright in a chair with full upper-body support. The arm on the dominant side was positioned so that the glenohumeral joint was in $70-80^{\circ}$ of flexion (sagittal plane) - a position that has shown an optimal infraspinatus activation [15] without compromising the subacromial space [16]. The elbow was kept in $90^{\circ}$ of flexion supported by an armrest. The participant was instructed to perform repeated external rotations of the shoulder in this position with resistance from a firm elastic band. When fatigue set in and the participant could no longer actively perform the exercise, the contralateral arm was used to assist the concentric phase of the movement and then resist the eccentric phase on the way back using the dominant arm only. Participants performed four sets of this exercise separated by 1-minute rests. In each set, participants continued until failure. Failure was defined when the participant 1) could not continue due to fatigue; 2) could not perform the exercise throughout the full range; or 3) could not remain in an upright position while performing the exercise. A 6-point Likert scale was used to assess the effect of the DOMS exercise protocol on the test day, where 0 represented a complete absence of pain; 1 represented minimal pain or discomfort in the shoulder; 2 represented a light, persistent shoulder pain or ache; 3 represented moderate shoulder pain when moving the shoulder or lifting objects; 4 represented severe discomfort that disturbed natural shoulder movement; and 5 represented strong shoulder pain, impeding all shoulder movement or function.

\section{Pressure Stimulation Intensities}

A handheld pressure algometer (Algometer, Somedic SenseLab, Sösdala [AQ6], Sweden) with a 1- $\mathrm{cm}^{2}$ probe was used to record PPTs at the infraspinatus muscle and to perform the suprathreshold stimulation. The stimulation site was located in a standardized manner using anatomical landmarks of the shoulder blade as described previously [7]. For PPTs, the applied force was progressively increased at a constant rate of $30 \mathrm{kPa} / \mathrm{s}$, and the participant indicated when the sensation changed from pressure to pain. The procedure was repeated three times, and the average value was extracted for data analysis. All participants received four suprathreshold pressure stimulations at different intensities relative to the individual PPT value: 1$)$ PPT value $+20 \%(\mathrm{PPT}+20 \%) ; 2)$ PPT value $+30 \%(\mathrm{PPT}+30 \%) ; 3)$ PPT value $+40 \%$ ( $\mathrm{PPT}+40 \%)$; and 4$)$ PPT value $+50 \%$ ( $\mathrm{PPT}+50 \%)$. To minimize the risk that increasing stimulation intensities would gradually cause greater test-related sensitization at the stimulation site, stimulations were applied randomly in a balanced manner in an ascending (low to high) or descending (high to low) order, where participants received either the highest $(\mathrm{PPT}+50 \%)$ or lowest $(\mathrm{PPT}+20 \%)$ stimulation first. All participants were blind to the stimulation sequence. To reduce the possibility of tissue damage and residual soreness, only one 60 -second stimulation was assessed for each stimulation intensity. To maintain consistency, each stimulation intensity was reached within 2 seconds. Following each stimulation, participants indicated the pain intensity from the stimulation using a verbal rating scale (VRS) from 0 to 10 , where $0=$ no pain and 10=worst pain imaginable. A 5 -minute break was given between each stimulation.

\section{Assessment of Pressure-Induced Pain Area and Extent}

The area of referred pain was quantified using a Navigate Pain software application (Navigate Pain, Aalborg [AQ7], Aalborg University, Denmark) [17]. Based on the pain drawing, the total size of the referred pain area (expressed as pixels) was extracted. The extent of referred pain was post-processed using MATLAB (MathWorks, Natick, MA) to calculate the distance from a reference point (ipsilateral earlobe) to the most distally located pixel drawn on the body chart. The distance was presented as a pain vector length, expressed as pixels, and was used to determine how extensively the pain referral spread down the arm. For these purposes, the distance from the earlobe to the most distant pain area on the posterior view of the body chart was extracted for data analysis.

Previous studies using a similar pain model have shown that stimulating the infraspinatus results in a large pain referral area $[18,19]$, with pressure stimulation inducing referred pain mainly on the posterior side of a body chart [7]. As the purpose of this study [AQ8] was to evaluate differences in pain referral between different stimulation intensities, only the pain referral area drawn on the posterior part of the body chart was extracted for data analysis. 


\section{Statistics}

Data were analyzed using a repeated-measures analysis of variance (ANOVA) or Friedman's ANOVA depending on the distribution of data, which was determined using the Shapiro-Wilk test. A log transformation was performed on data that did not pass the normality tests before choosing the statistical method. Parametric data were expressed as means ( \pm standard deviation), whereas nonparametric data were expressed as median (interquartile range). For post hoc comparisons, the Newman-Keuls test (parametric data) or the Wilcoxon paired test with a Bonferroni correction (nonparametric data) were used. For significant findings, a correlation analysis (Pearson's correlation coefficient or Spearman's rank-order correlation, depending on normality) was used to assess the relationship between pain intensity (VRS) and pain referral (area in pixels and pain vector length). Likewise, the relationship between the size of the pain area (pixels) and extent (pain vector length) was investigated.

The effect of sequence was investigated prior to pooling data using a mixed-model ANOVA, where order (increasing or decreasing order of stimuli) was set as a fixed factor and the stimulation intensities were repeated factors. Similarly [AQ9], gender was investigated prior to pooling the data, as women report referred pain more frequently and their areas of pain are larger as compared with men $[9,20]$.

To account for the large variability in pain referral patterns from a standardized stimulation as seen in previous experimental pain studies [7, 10,14], the coefficient of variation was calculated to determine any potential differences in the variation in size of the pain area, the pain vector length, and the pressure-induced pain intensity between the different stimulation intensities (percentage of PPT). A $P$ value less than 0.05 was considered statistically significant.

Due to the exploratory nature of this study, the achieved power was calculated post hoc using G*Power (version 3.1.9.4, University of Düsseldorf, Düsseldorf [AQ10]) [21].

\section{Results}

A full data set from 22 participants (age, 30 years [23-44 years]; height, $172 \mathrm{~cm}$ [154-186 cm]; and weight, $67 \mathrm{~kg}$ [46-90 kg]) was available for data analysis. The pain intensity (VRS) and pain area passed the normality test (pain area following log transformation), whereas pain vector length and DOMS symptoms did not. An investigation of sequence (increasing or decreasing order of stimuli) showed no effect of order of stimuli (mixed-model ANOVA: $P>0.05)$. Moreover, no effects of gender were found. Data were therefore pooled prior to analysis.

The median Likert score of DOMS on day 2 was 3 (2-3), with one participant reporting no DOMS. The participants' data were nevertheless included in the data set in accordance with previous procedures [7].

\section{Pressure-Induced Area and Extent of Referred Pain}

The pressure stimulation caused an extensive pain referral (Figure 1), with a significant interaction found in the size of the pain area between the different pressure intensities used to induce referred pain (repeated-measures ANOVA [AQ11]: $F[3,63]=3.8, P<0.03)$. Here, the pain area induced at PPT $+20 \%$ was significantly smaller than that at $\mathrm{PPT}+30 \%($ Newman-Keuls, NK [AQ12]: $P<0.02)$, PPT $+40 \%(\mathrm{NK}: P<0.05)$, and PPT $+50 \%(\mathrm{NK}: P<0.04)(\mathrm{Ta}-$ ble 1). No significant difference in pain area was found when comparing the other stimulation intensities (NK: $P>0.05)$. A significant effect for pressure intensity was found for pain vector length $\left(\chi^{2}[3]=15.5, P<0.001\right)$, where the PPT $+50 \%$ intensity extended farther away from the stimulus than PPT $+30 \%$ (Wilcoxon: $P<0.002)($ Table 1 ).

Figure 1. Overlays of each pain drawing associated with the different pressure intensities showing the pressure-induced pain referral pattern, as viewed from anterior and posterior perspectives. The overlays of pain drawings are presented as overlays of all the original individual pain drawings for each stimulation intensity. The darkest regions and lightest shading indicate the areas of maximum and minimum overlap, respectively, for the group at PPT $+20 \%$, PPT $+30 \%$, PPT $+40 \%$, and $\mathrm{PPT}+50 \%$. PPT $=$ pressure pain threshold. [AQ36] 


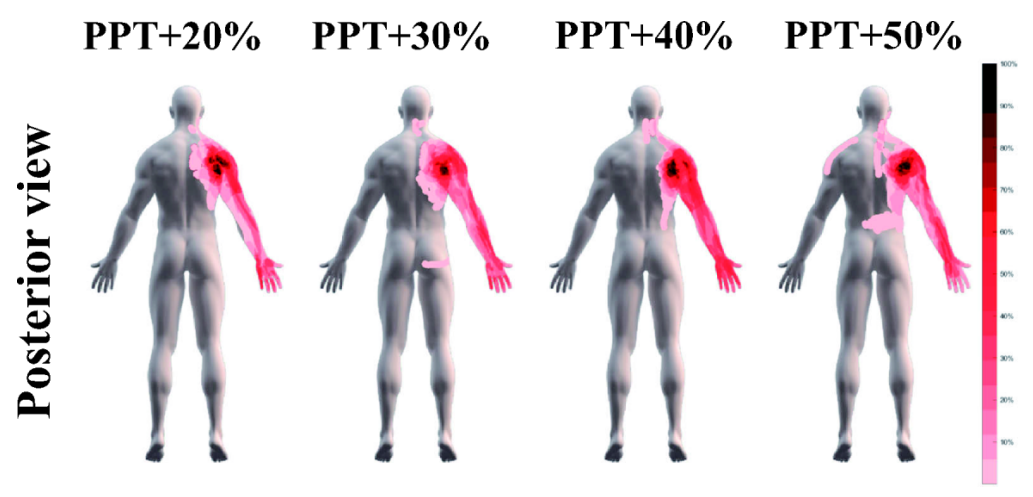

Table 1. Mean $( \pm \mathrm{SD})$ size of the area (in pixels), pain vector length (log-transformed values), and pain intensity on a verbal rating scale following tonic pressure stimulation

\begin{tabular}{|l|l|l|l|l|}
\hline PPT Infraspinatus Stimulation In- & $\begin{array}{l}\text { Size of the Area (Coefficient } \\
\text { tensity }\end{array}$ & $\begin{array}{l}\text { Pain Vector Length (Co- } \\
\text { officient of Variation) }\end{array}$ & $\begin{array}{l}\text { Pain (Coefficient of } \\
\text { Variation) }\end{array}$ \\
\hline \multirow{3}{*}{$383.0 \pm 141.8$} & PPT $+20 \%$ & $2,623 \pm 3,002(114.5)$ & $364 \pm 256(70)$ & $5.9 \pm 1.8(30.5)$ \\
\cline { 2 - 5 } & PPT $+30 \%$ & $3,972 \pm 4,069^{*}(102.4)$ & $266 \pm 144^{\dagger}(54)$ & $6.4 \pm 1.3(20.3)$ \\
\cline { 2 - 5 } & PPT $+40 \%$ & $4,270 \pm 4,452^{*}(104.3)$ & $262 \pm 133(51)$ & $6.8 \pm 1.5^{*}(22.1)$ \\
\cline { 2 - 5 } & PPT $+50 \%$ & $3,924 \pm 4,015^{*}(102.3)$ & $404 \pm 233^{\dagger}(58)$ & $7.3 \pm 1.8^{\ddagger}(24.7)$ \\
\hline
\end{tabular}

$\mathrm{SD}=$ standard deviation; $\mathrm{PPT}=$ pressure pain threshold [AQ34].

* Newman-KeulsNot known [AQ35]—different compared with PPT $+20 \%$.

$\dagger$ Wilcoxon (Bonferroni corrected) difference between the indicated stimulation intensities.

$\$$ Not knownNewman-Keuls - different from all other stimulation intensities.

A significant effect was found for pain intensity between the four different pressure intensities (ANOVA: $F[3,63]$ $=8.1, P<0.0001)$, where the PPT $+50 \%$ intensity caused greater pain than all the other pressure intensities (NK: $P<0.05)$ and $\mathrm{PPT}+40 \%$ caused greater pain than $\mathrm{PPT}+20 \%(\mathrm{NK}: P<0.02)($ Table 1$)$.

An investigation of the association among the size (number of pixels), extent (vector length), and the pain intensity (VRS) demonstrated no relationship between pain intensity and size (Spearman's [AQ13] $R_{S}=-0.018, P<0.87$ ) or extent (Spearman's $R_{S}=-0.029, P<0.79$ ). A significant positive correlation between the size (number of pixels) and extent (pain vector length) was found (Pearson's $r=0.55, P<0.0001$ ).

The coefficient of variation indicated that the greatest variability was seen in the lowest stimulation intensity (PPT $+20 \%$ ) for the size of the referred pain area, the pain vector length, and the perceived pain (Table 1). However, the coefficients were comparable between the other stimulation intensities.

\section{Discussion}

The results from the current study indicate that a sustained pressure stimulation of $30 \%$ above the PPT value is optimal in terms of inducing the largest pain referral area with the least amount of pressure. Moreover, the relationship between pressure stimulation intensity and the extent of the pain area does not seem to be linear. The potential value of this information will be discussed in the following sections.

\section{Pressure-Induced Pain Referral Increases with Higher Pressure Intensities}

Using pressure stimulation to assess pain referral patterns is a safe and noninvasive method used to explore the sensitivity of the pain system. Increasing the pressure stimulation intensity from $20 \%$ to $30 \%$ above the PPT results in 
a significantly larger pain referral area, but applying pressure at greater intensities does not seem to increase the size of the pain area despite increases in pain intensity (Table 1).

For research purposes, assessing pain referral in clinical groups may seem important, as it may help to establish whether the condition is related to increased sensitivity of central pain mechanisms [22]. The invasiveness of methods traditionally used to induce referred pain [AQ14], such as chemical or electrical stimulations [23], may, however, not be feasible due to, for example, patients' fear of needles and the accuracy needed to stimulate the desired structure. Using a stimulation intensity of $30 \%$ above the PPT has been used to assess pain referral patterns in the lower leg [8], and the current findings suggest that this intensity may be ideal for inducing referred pain if weighing the nonsignificant increase in pain against the significant increase in pain referral.

In this study, the participants indicated the size and location of the pain area by drawing on an electronic body chart (Navigate Pain). The drawn area can quickly be extracted as the number of colored pixels within the body chart. Without a visual representation, information regarding the extent of pain spreading away from the originating nociceptive stimulus will be missing. Pain can be referred by stimulating both the somatic and neural structures with a considerable overlap with regard to location of symptoms. This overlap can be seen by comparing the findings of, for example, Palsson and Graven-Nielsen [24] with the recent findings of Furman and Johnson [25]. Therefore, assessing the distance between the site farthest from the nociceptive stimulus was extracted and presented as the pain vector length. Combining these two values (size and pain vector length) into one number instead of presenting them separately (Table 1) would be desirable. The correlation analysis indicated a significant association between pain size and pain vector length, but only $30 \%$ of the variability in one variable can be explained by the changes in the other (based on a correlation of $r=0.55$ ). Therefore, the two measurements (size in pixels and pain vector length) seem to present unique characteristics of referred pain that cannot be represented by only extracting one of the variables. This can be seen in the data where, for example, the lowest $(\mathrm{PPT}+20 \%)$ and highest $(\mathrm{PPT}+50 \%)$ stimulation intensities seem to induce the longest pain vector lengths. Further investigations into the unique contributions of size in pixels and pain vector length are warranted to understand referred pain.

Interestingly, no associations were found between the pain intensity and the size or extent of the pain referral, although similar associations have previously been reported [1,9]. The reason for this discrepancy is unclear but may relate to the high variability seen in the PPT values, pain intensity, and pain referral patterns (Table 1).

\section{Methodological Considerations and Limitations}

As shown in Table 1, the variability in pain referral is considerable, indicating that the response to suprathreshold pressure stimulation is different between individuals who receive the same relative stimulus. When considering the coefficient of variation, however, the variability is greater at PPT $+20 \%$ than at the other stimulation intensities for all outcome measures (area, pain vector length, and self-reported pain). The variability seen in this study is similar to what has been demonstrated in previous studies using both chemical and mechanical stimulations to induce referred pain $[7,10]$ and is important to consider, as it inevitably questions the value of using pain referral as a stand-alone diagnostic tool for assessing the sensitivity of central pain mechanisms. Indeed [AQ15], it has been recommended to integrate referred pain areas with a battery of other quantitative pain measurements to assess pain mechanisms [26].

PPTs were only assessed after eliciting DOMS in the shoulder muscles. Previous studies have [AQ16] shown that DOMS reduces PPT, increases the probability of pain referral, and enhances pain referral in comparison with preDOMS conditions $[7,13,14]$. Considering that DOMS is transient and is primarily used as a model of musculoskeletal pain, patterns of pain referral were only investigated for suprathreshold pressure stimulations during this condition. In this way, stimulations would require less force to induce patterns of referred pain, reduce the possibility of residual soreness, and provide information about a sensitized system.

The current protocol used a stimulation paradigm consisting of a 60 -second-long pressure stimulation on the infraspinatus muscle in accordance with previous procedures [11]. This duration was chosen because it is known that the spreading of pain is delayed with regard to the onset of the primary nociceptive stimulus [27, 28]. A stimulation of a shorter duration would be feasible, especially when assessing people in pain. However, a stimulation of a shorter duration (e.g., 5 seconds) results in a smaller area of pain referral than if the stimulation lasts longer [7]. A study designed for capturing the temporal development of referred pain would be useful for determining the optimal duration of stimulation. 
The infraspinatus muscle was chosen to determine the effects of different pressure pain on referred pain despite the tibialis anterior muscle being commonly investigated $[8,10]$. For the tibialis anterior, the protocols use relatively low stimulation intensities $(\mathrm{PPT}+20 \%$ and $\mathrm{PPT}+30 \%)$. The main reason [AQ17] for the lower intensities is that the tibialis anterior muscle is located in the anterior compartment of the lower leg. When applying pressure to the tibialis anterior, the force is more easily transferred into the deeper layers of the compartment, thus requiring greater force to reach the PPT. In contrast [AQ18], the infraspinatus muscle is relatively thin, with the scapula lying deep within the muscle, making the muscle and location ideal for this study. As this study used tonic stimulations of up to $50 \%$ above the PPT, the infraspinatus site would reduce the possibility of residual soreness at higher stimulation intensities. Moreover, it has previously been shown that painful stimulation of the infraspinatus muscle can evoke referred pain in the majority (up to $83 \%$ ) of healthy participants [18].

Using the binary stimulation order - either low-to-high stimulation or high-to-low stimulation — did not seem to affect the outcome. Nevertheless, it introduces a risk of expectation affecting the outcome (self-reported area of pain referral). Likewise, even though 5 minutes passed between each stimulation intensity, it is not possible to rule out the possibility of residual pain influencing the outcome. To minimize the potential influence of order, randomizing the order of stimulation intensities might be a feasible option for future studies. However, the current results do not support a progressive summation of pain (area and extent) due to increasing or decreasing pressure pain. The finding that [AQ19] the pressure pain-evoked area (e.g., at PPT $+30 \%$ ) did not differ from that of the two higher pressure pain intensities counterindicates a summation effect.

Although previous studies indicate that women more often experience referred pain than men from a standardized stimulus $[9,20]$, no gender differences were observed in this study. It is likely that the sample size $(\mathrm{N}=22,10$ women $)$ was too small to capture gender differences, considering that this has previously been demonstrated in a larger sample $(\mathrm{N}=72)$ [20]. The choice of muscle and stimulation type and intensities differs in this study as compared with others. The presence of DOMS in the infraspinatus muscle leads to patterns of evoked referred pain that are consistent with those of clinical populations [18, 29, 30]. Additionally, the infraspinatus muscle is generally smaller, more superficial, and may exhibit fewer anatomical differences, such as size, in the tibiablis anterior between sexes. It would be interesting to investigate whether increasing stimulation intensities causes gender-specific responses.

The current findings may be interesting to consider through the lens of pain referral from manual stimulation of pain-sensitive musculoskeletal structures. It has been suggested that a taut band, a hypersensitive spot, and referred pain are the diagnostic criteria for a musculoskeletal trigger point [31]. Although no attempt was made to determine the presence of a taut band - bearing in mind the difficulty of doing so in a valid manner [32] — the participants reported pain in the shoulder on the test day and experienced referred pain from pressure stimulation. In this study, the pain area increased with higher pressure values where, by merely increasing the stimulation intensity by $10 \%$ (from $20 \%$ to $30 \%$ above the PPT), the pain area became significantly larger. The current findings cannot be directly applied to pre-DOMS conditions or extrapolated to clinical populations, as the participants were healthy individuals experiencing transient pain created by an exercise-induced muscle soreness protocol. Nevertheless, the findings presented herein may have value in a clinical setting, where it is more difficult to standardize the pressure applied manually, and indicate that clinicians should be careful when interpreting the response to their manual assessment and treatment.

\section{Conclusion}

This study compared the pain referral patterns from suprathreshold pressure stimulation at four different intensities above the PPT at the infraspinatus muscle. The findings indicate that a stimulation intensity at $30 \%$ above the PPT value is a feasible option when considering the size of the pain referral pattern and the perceived pain.

\section{Acknowledgments}

The authors wish to thank the volunteers for their participation in the study.

\section{References}

1. Arendt-Nielsen L, Svensson P. Referred muscle pain: Basic and clinical findings. Clin J Pain 2001;17(1):11-9.

2. Cooper G, Bailey B, Bogduk N. Cervical zygapophysial joint pain maps. Pain Med 2007;8(4):344-53.

3. Kosek E, Januszewska A. Mechanisms of pain referral in patients with whiplash associated disorder. Eur J Pain 2008;12(5):650-60. 
4. O’Neill S, Manniche C, Graven-Nielsen T, Arendt-Nielsen L. Generalized deep-tissue hyperalgesia in patients with chronic low-back pain. Eur J Pain 2007;11(4):415-20. [AQ20]

5. Palsson TS, Hirata RP, Graven-Nielsen T. Experimental pelvic pain impairs the performance during the active straight leg raise test and causes excessive muscle stabilization. Clin J Pain 2015;31(7):642-51.

6. O’Neill S, Graven-Nielsen T, Manniche C, Arendt-Nielsen L. Ultrasound guided, painful electrical stimulation of lumbar facet joint structures: An experimental model of acute low back pain. Pain 2009;144(1-2):76-83.

7. Domenech-Garcia V, Palsson TS, Herrero P, Graven-Nielsen T. Pressure-induced referred pain is expanded by persistent soreness. Pain 2016;157(5):1164-72.

8. Gibson W, Arendt-Nielsen L, Graven-Nielsen T. Referred pain and hyperalgesia in human tendon and muscle belly tissue. Pain 2006;120(1-2):113-23.

9. Graven-Nielsen T. Fundamentals of muscle pain, referred pain and deep tissue hyperalgesia. Scand J Rheumatol Suppl 2006;122:1-43. [AQ21]

10. Palsson TS, Boudreau SA, Krebs HJ, Graven-Nielsen T. Experimental referred pain extends toward previously injured location: An explorative study. J Pain 2018;19(10):1189-200. [AQ22]

11. Doménech-García V, Skuli Palsson T, Boudreau SA, Herrero P, Graven-Nielsen T. Pressure-induced referred pain areas are more expansive in individuals with a recovered fracture. Pain 2018;159(10):1972-9.

12. Slater H, Arendt-Nielsen L, Wright A, Graven-Nielsen T. Sensory and motor effects of experimental muscle pain in patients with lateral epicondylalgia and controls with delayed onset muscle soreness. Pain 2005;114(1-2):118-30.

13. Gibson W, Arendt-Nielsen L, Graven-Nielsen T. Delayed onset muscle soreness at tendon-bone junction and muscle tissue is associated with facilitated referred pain. Exp Brain Res 2006;174(2):351-60.

14. Doménech-García V, Palsson TS, Boudreau SA, Herrero P, Graven-Nielsen T. Pressure-induced referred pain areas are more expansive in individuals with a recovered fracture. Pain 2018;159(10):1972-9. [AQ23]

15. Ha S-M, Kwon O-Y, Cynn H-S, Lee W-H, Kim S-J, Park K-N. Selective activation of the infraspinatus muscle. J Athl Train 2013;48(3):346-52.

16. Roberts CS, Davila JN, Hushek SG, Tillett ED, Corrigan TM. Magnetic resonance imaging analysis of the subacromial space in the impingement sign positions. J Shoulder Elbow Surg 2002;11(6):595-9.

17. Boudreau SA, Badsberg S, Christensen SW, Egsgaard LL. Digital pain drawings: Assessing touch-screen technology and 3D body schemas. Clin J Pain 2016;32(2):139-45. [AQ24]

18. Leffler AS, Kosek E, Hansson P. Injection of hypertonic saline into musculus infraspinatus resulted in referred pain and sensory disturbances in the ipsilateral upper arm. Eur J Pain 2000;4(1):73-82. [AQ25]

19. Kosek E, Hansson P. The influence of experimental pain intensity in the local and referred pain area on somatosensory perception in the area of referred pain. Eur J Pain 2002;6(6):413-25. [AQ26]

20. Frey Law LA, Sluka KA, McMullen T, Lee J, Arendt-Nielsen L, Graven-Nielsen T. Acidic buffer induced muscle pain evokes referred pain and mechanical hyperalgesia in humans. Pain 2008;140(2):254-64.

21. Faul F, Erdfelder E, Lang A-G, Buchner AG. GPower 3: A flexible statistical power analysis program for the social, behavioral, and biomedical sciences. Behav Res Methods 2007;39(2):175-91. [AQ27]

22. Arendt-Nielsen L, Graven-Nielsen T. Central sensitization in fibromyalgia and other musculoskeletal disorders. Curr Pain Headache Rep 2003;7(5):355-61.

23. Graven-Nielsen T, Arendt-Nielsen L. Assessment of mechanisms in localized and widespread musculoskeletal pain. Nat Rev Rheumatol 2010;6(10):599-606. [AQ28]

24. Palsson TS, Graven-Nielsen T. Experimental pelvic pain facilitates pain provocation tests and causes regional hyperalgesia. Pain 2012;153(11):2233-40. 
25. Furman MB, Johnson SC. Induced lumbosacral radicular symptom referral patterns: A descriptive study. Spine J 2019;19(1):163-70.

26. Arendt-Nielsen L. Central sensitization in humans: Assessment and pharmacology. Handbook Exp Pharmacol 2015;227:79-102. [AQ29]

27. Laursen RJ, Graven-Nielsen T, Jensen TS, Arendt-Nielsen L. Quantification of local and referred pain in humans induced by intramuscular electrical stimulation. Eur J Pain 1997;1(2):105-13. [AQ30]

28. Graven-Nielsen T, Arendt-Nielsen L, Svensson P, Jensen TS. Stimulus-response functions in areas with experimentally induced referred muscle pain—A psychophysical study. Brain Res 1997;744(1):121-8.

29. Koelbaek Johansen M, Graven-Nielsen T, Schou Olesen A, Arendt-Nielsen L. Generalised muscular hyperalgesia in chronic whiplash syndrome. Pain 1999;83(2):229-34.

30. Bron C, Dommerholt J, Stegenga B, Wensing M, Oostendorp RA. High prevalence of shoulder girdle muscles with myofascial trigger points in patients with shoulder pain. BMC Musculoskelet Disord 2011;12(1):139. [AQ31]

31. Fernández-de-Las-Peñas C, Dommerholt J. International consensus on diagnostic criteria and clinical considerations of myofascial trigger points: A Delphi study. Pain Med 2018;19(1):142-50. [AQ32]

32. Quintner JL, Bove GM, Cohen ML. A critical evaluation of the trigger point phenomenon. Rheumatology

2015;54(3):392-9. [AQ33]

\section{AUTHOR QUERIES}

Query: AQ1: Please check all author names and affiliations. Please check that author surnames have been identified by a pink background in the PDF version and by green text in the HTML proofing tool version (if applicable). This is to ensure that forenames and surnames have been correctly tagged for online indexing.

Author Response: Pablo Herrero has changed his affiliation to Faculty of Health Sciences, University of Zaragozapherrero@unizar.esThis affiliation can come number 7Elisabeth Bravo Esteban's surname needs to be changed to: Elisabeth Bravo Esteban-HerrerosOther names and affiliations are correct

Query: AQ2: Please provide the city name for the Universidad de Castilla la Mancha. Author Response: Ciudad real

Query: AQ3: Please confirm that the information for the corresponding author is correct as written.

Author Response: Accept

Query: AQ4: If your manuscript has figures or text from other sources, please ensure you have permission from the copyright holder. For any questions about permissions, contact jnls.author.support@oup.com.

Author Response: Accept

Query: AQ5: The sentence "However, although the parameters of hypertonic saline stimulation (e.g., volume, concentration) are well established [9], the optimal parameters for pressure stimulation are not known" was revised for clarity. Please confirm that it is correct as written and does not change your intended meaning.

Author Response: Accept

Query: AQ6: Please provide the manufacturer city name for Somedic SenseLab.

Author Response: Answered within text

Query: AQ7: Please provide the manufacturer city name for Navigate Pain.

Author Response: Answered within text

Query: AQ8: The sentence "As the purpose of this study was to evaluate differences in pain referral between different stimulation intensities, only the pain referral area drawn on the posterior part of the body chart was extracted for data analysis" was revised for clarity. Please confirm that it is correct as written and does not change your intended meaning.

Author Response: Accept 
Query: AQ9: The sentence "Similarly, gender was investigated prior to pooling the data, as women report referred pain more frequently and their areas of pain are larger as compared with men $[9,20]$ " was revised for clarity. Please confirm that it is correct as written and does not change your intended meaning.

Author Response: Accept

Query: AQ10: Please provide the manufacturer city and country or state name for G*Power.

Author Response: Answered within text

Query: AQ11: Please confirm that "RM" stands for "repeated measures." This was spelled out for clarity.

Author Response: Accept

Query: AQ12: Please spell out "NK" on first mention.

Author Response: Answered within text

Query: AQ13: Please confirm that the equations for Spearman's Rs are correct as written.

Author Response: Accept

Query: AQ14: Please confirm that "RP" stands for "referred pain." This was spelled out throughout for clarity.

Author Response: Accept

Query: AQ15: The sentence "Indeed, it has been recommended to integrate referred pain areas with a battery of other quantitative pain measurements to assess pain mechanisms [26]" was revised for clarity. Please confirm that it is correct as written and does not change your intended meaning.

Author Response: Accept

Query: AQ16: The sentence "Previous studies have shown that DOMS reduces PPT, increases the probability of pain referral, and enhances pain referral in comparison with pre-DOMS conditions [7,13,14]" was revised for clarity.

Please confirm that it is correct as written and does not change your intended meaning.

Author Response: Accept

Query: AQ17: The sentence "The main reason for the lower intensities is that the tibialis anterior muscle is located in the anterior compartment of the lower leg" was revised for clarity. Please confirm that it is correct as written and does not change your intended meaning.

Author Response: Accept

Query: AQ18: The sentence "In contrast, the infraspinatus muscle is relatively thin, with the scapula lying deep within the muscle, making the muscle and location ideal for this study" was revised for clarity. Please confirm that it is correct as written and does not change your intended meaning.

Author Response: Accept

Query: AQ19: The sentence "The finding that the pressure pain-evoked area (e.g., at PPT+30\%) did not differ from that of the two higher-pressure pain intensities counterindicates a summation effect" was revised for clarity. Please confirm that it is correct as written and does not change your intended meaning.

Author Response: Accept

Query: AQ20: Please note that original references 4, 8, and 10 are duplicates of references 14,16 , and 23. The duplicate references have been removed and the subsequent references have been renumbered accordingly.

Author Response: Accept

Query: AQ21: Please provide the issue number for Reference 9 (Graven-Nielsen) if possible.

Author Response: there is no issue number

Query: AQ22: Please confirm that Reference 10 (Palsson et al.) is correct as written.

Author Response: Accept

Query: AQ23: Please confirm that Reference 14 (Doménech-García et al.) is correct as written.

Author Response: Accept

Query: AQ24: Please confirm that Reference 17 (Boudreau et al.) is correct as written.

Author Response: Accept

Query: AQ25: Please confirm that Reference 18 (Leffler et al.) is correct as written. 


\section{Author Response: Accept}

Query: AQ26: Please confirm that Reference 19 (Kosek and Hansson) is correct as written. Author Response: Accept

Query: AQ27: Please confirm that Reference 21 (Faul et al.) is correct as written. Author Response: Accept

Query: AQ28: Please confirm that Reference 23 (Graven-Nielsen and Arendt-Nielsen) is correct as written. Author Response: Accept

Query: AQ29: Please provide the issue number for Reference 26 (Arendt-Nielsen) if possible.

Author Response: There is no issue number

Query: AQ30: Please confirm that Reference 27 (Laursen et al.) is correct as written. Author Response: Accept

Query: AQ31: Please confirm that Reference 30 (Bron et al.) is correct as written. Author Response: Accept

Query: AQ32: Please confirm that Reference 31 (Fernández-de-Las-Peñas and Dommerholt) is correct as written. Author Response: Accept

Query: AQ33: Please confirm that Reference 32 (Quintner et al.) is correct as written. Author Response: Accept

Query: AQ34: Please confirm that the footnotes for Table 1 are correct as written. Author Response: Accept

Query: AQ35: Please confirm that "NK" stands for "not known" in the footnotes for Table 1. This was spelled out for clarity.

Author Response: Answered within text

Query: AQ36: These figures are currently intended to appear online in colour and black and white in print. Please reword the legend/text to avoid using reference to colour. Alternatively, please let us know if you wish to pay for print colour reproduction or to have both versions in black and white. The standard charge for colour reproduction in print is $£ 350 / e 525 / \$ 600$ per figure. Please check the black and white versions at the end of the paper and contact us if you have any concerns. If there are no color figures in your article, please ignore this query

Author Response: The darkest to lightest shading works fine in black and white. We do not wish to pay for color versions 